\title{
Rapamycin ameliorates brain damage and maintains mitochondrial dynamic balance in diabetic rats subjected to middle cerebral artery occlusion
}

\section{Changchun Hei}

Ningxia Medical University

\section{Yujia Zhou}

General Hospital of Ningxia Medical University

Chenyang Zhang (D 820692080@qq.com)

Ningxia Medical University https://orcid.org/0000-0001-6264-3683

\section{Furong Gao}

Medical Security Bureau of Jiaxiang County

\section{Meiling Cao}

General Hospital of Ningxia Medical University

\section{Shilin Yuan}

General Hospital of Ningxia Medical University

\section{Yixin Qin}

General Hospital of Ningxia Medical University

\section{P. Andy Li}

North Carolina Central University

\section{Xiao Yang}

General Hospital of Ningxia Medical University https://orcid.org/0000-0003-3065-7225

\section{Research Article}

Keywords: Rapamycin, Diabetes, Cerebral ischemia, Mitochondrial dynamics, Mitochondrial fission and fusion, SIRT3

Posted Date: February 17th, 2022

DOI: https://doi.org/10.21203/rs.3.rs-1358085/v1

License: (9) This work is licensed under a Creative Commons Attribution 4.0 International License. Read Full License 


\section{Abstract}

Objective To investigate the effect of rapamycin on mitochondrial dynamic balance in diabetic rats subjected to cerebral ischemia-reperfusion injury.

Methods Sprague Dawley male rats were treated with high fat diet combined with streptozotocin injection to construct diabetic model in rats. Transient middle cerebral artery occlusion (MCAO) of 2 hours was induced and the brains were harvested after 1 and 3 days of reperfusion. Rapamycin was injected intraperitoneally for 3 days prior to and immediately after operation, once a day. The neurological function was assessed, infarct volumes were measured and histological outcome were examined. The total protein of hippocampus was extracted and Western blotting were performed to detect the levels of mTOR, mitochondrial dynamin related proteins (DRP1, p-DRP1, OPA1), SIRT3, and Nix/BNIP3L.

Results Diabetes worsened the neurological function performance, enlarged infarct size and increased ischemic neuronal cell death. The increased damage was associated with elevations of p-mTOR, p-S6, and p-DRP1; and suppressions of SIRT3 and Nix/BNIP3L. Rapamycin ameliorated diabetes-enhanced ischemic brain damage and reversed the biomarker alterations caused by diabetes.

Conclusion High glucose activated mTOR pathway and caused mitochondrial dynamics toward fission. The protective effect of Rapamycin against diabetes-enhanced ischemic brain damage was associated with inhibiting mTOR pathway, redressing mitochondrial dynamic imbalance, and elevating SIRT3 and Nix/BNIP3L expression.

\section{Introduction}

There are mainly two types of stroke: ischemic and hemorrhagic. Ischemic stroke accounts for $69.6 \%-70.8 \%$ of all strokes and it is one of the main causes of disability and death in adults. Ischemic stroke has become a public health threat (Collaborators 2020;Li et al. 2019). Studies have found that many risk factors are significantly associated with the progression and prognosis of ischemic stroke, including controllable factors such as obesity, diabetes, heart disease, hypertension, high cholesterol, smoking, and uncontrolled factors such age, gender, and family history of stroke (Patnaik et al. 2019). As a recognized independent risk factor for ischemic stroke, diabetes significantly increase the incidence and mortality of stroke (Li et al. 2020). It has been reported that the incidence of ischemic stroke in diabetic patients is 1.5-2 times higher than that in non-diabetic patients; In addition, the total mortality of stroke patients with diabetes is 4-5 times higher than that of non-diabetic patients (Dong et al. 2018;Rinkel et al. 2020). A large number of animal experimental studies and our previous studies have also verified that hyperglycemia can increase the infarct size after ischemic stroke and affect the prognosis of stroke (Liu et al. 2016;Yang et al. 2015). However, the specific pathological mechanisms about how hyperglycemia aggravating cerebral ischemia-reperfusion injury remain unclear, but may include lactic acid accumulation, impaired calcium homeostasis, increased production of reactive oxygen and nitrogen 
species, activation of apoptotic pathways, initiation of neuroinflammation, excessive mitochondrial fission, and activation of autophagy (Hei et al. 2017).

The dynamic balance of mitochondria refers to the process of alternating and cycling the dynamics of mitochondrial fission and fusion. Through continuous fission and fusion, the morphology, quantity and distribution of mitochondria are under control (Chen et al. 2020). The imbalance of fission and fusion is involved in the pathogenesis of many diseases. For example, in the model of cerebral ischemia, it is found that hypoxia increases the production of reactive oxygen species (ROS), increases the expression of mitochondrial mitogen protein: dynamin-related protein-1 (DRP1) and mitochondrial fission 1 (Fis1), decreases the expression of mitochondrial fusion proteins: optic atrophy-1 (OPA1) and mitochondrial fusion proteins (Mfn2), resulting in increase of apoptotic cells and enlargement of infarct area in mouse brain tissue. High glucose further aggravates the above alterations. Inhibition of mitochondrial fission ameliorates tissue damage caused by hypoxia and high glucose (He et al. 2020). Mitochondrial fissionfusion imbalance may also be involved in diabetes-aggravated ischemia-reperfusion injury. When the harmful factors of the external environment affected the cell, the mitochondria change its dynamic balance. Mitochondrial fission produces two sub-mitochondria: a polarized and a depolarized one. The polarized mitochondria reintegrated into the mitochondrial network, while the depolarized one is cleared by mitochondrial autophagy (Guo et al. 2013). Excessive mitochondrial fission leads to reduced energy production, mitochondrial fragmentation and cell death. Mammalian rapamycin target protein (mTOR) is a serine/threonine protein kinase that regulates many biological processes including protein synthesis, cell growth, ribosome production and autophagy. As the main regulator of cell metabolism, mTOR senses and integrates a variety of information inputs from upstream signals, such as growth factors, nutrients, amino acids, hunger and hypoxia. It plays a key role in regulating cell growth, proliferation and movement (Rodriguez-Rodriguez et al. 2019;Yang et al. 2020). Rapamycin, a specific inhibitor of mTOR, is a macrolide antibiotic with immunosuppressive and anti-proliferative effects. Although the role of mTOR in cerebral ischemia is controversial, more and more evidence showed that rapamycin may have a protective effect on cerebral ischemia. For example, we have previously reported that that rapamycin (6 $\mathrm{mg} / \mathrm{kg}$ ) significantly improved ischemic injury after global cerebral ischemia-reperfusion in rats under both normoglycemic and hyperglycemic conditions (Guo et al. 2013;Yang et al. 2015).

The objective of this study was to investigate the effect of rapamycin on mitochondrial dynamic balance in diabetic rats. To this end, we used rat model of middle cerebral artery ischemia-reperfusion (MCAO), which closely simulates the pathophysiological process of clinical cerebral stroke, and pretreated the animals with rapamycin $(3 \mathrm{mg} / \mathrm{kg})$. The results showed that rapamycin reduced infarct volume and improved neurofunctional recovery in diabetic animals after ischemia and reperfusion injury. This neuroprotective effect was associated with suppression of mTOR signaling, rebalance of mitochondrial dynamic imbalance caused by diabetic hyperglycemia.

\section{Materials And Methods}

\subsection{Experimental animals and solution preparation}


A total of 117 SPF male SD rats at 8 to 10 weeks old were purchased from the Experimental Animal Center of Ningxia Medical University, with a body weight of (230-280) g. The animal care, use and surgical procedures were was approved by the Experimental Animal Ethics Committee of Ningxia Medical University and consistent with the health guidelines of the National Institute of Health for the Care and Use of Laboratory Animals. Rapamycin stock solution was prepared by dissolving 100 mg rapamycin (LC Laboratories, USA, V900930) in $5 \mathrm{ml}$ of $100 \%$ ethanol $(20 \mathrm{mg} / \mathrm{mL})$ and stored at $-20^{\circ} \mathrm{C}$. Before

intraperitoneal injection, the stock solution was diluted with 5\% Tween-80 and 5\% polyethylene glycol 400 to a working solution with a final concentration of $4 \%$ ethanol.

\subsection{Animal group}

The animals were randomly divided into three groups: Normal blood glucose group (NG, $n=39)$, diabetic group (DM, $n=39$ ) and Diabetic rats treated with rapamycin (DMR, $n=39$ ). Each group was further divided into three subgroups: sham operation group (Sham), ischemia with 1 day reperfusion (NG1d, DM1d, and DMR1d) and ischemia with 3-day reperfusion (NG3d, DM3d, and DMR3d). Diabetes mellitus was induced in animals fed with high fat diet for one month, and then intraperitoneally injected with $2 \%$ streptozotocin (STZ, $50 \mathrm{mg} / \mathrm{kg}$ ). The blood glucose levels were measured at 3 and 7 days post-STZ injection and animals whose blood glucose value was more than $16.7 \mathrm{mmol} / \mathrm{L}$ in both readouts were considered successful induction of diabetic model and incorporated into diabetic group. In rapamycin treatment group, rapamycin was injected intraperitoneally to diabetic rats for 3 days before operation and immediately after operation. Four rats in each group were stained with 2-Magne3-5fluorophenyltetrazoliumchloride (TTC) to observe the infarct volume, 5 rats in each group were perfused and fixed in 4\% paraformaldehyde for pathological examination and immunohistochemistry, and 4 rats in each group were used for Western blotting.

\subsection{Cerebral Ischemic model}

Animals were fasted overnight with free access to water before surgery. The animals were inhaled $3.0 \%$ isoflurane in a gas mixture of $\mathrm{N} 2 \mathrm{O}$ and $\mathrm{O} 2$ (70:30) to induce anesthesia and maintain at concentration of 1.0-2.0\% during the operation. The rats were fixed on the operating table supine and shaved off the hair on neck. A $1.5 \sim 2.0 \mathrm{~cm}$ incision was made in ventrocervical skin. MCAO model was induced by suture occlusion. Briefly, after isolations of the right common carotid, external carotid and internal carotid arteries, the internal carotid artery was temporarily ligated to block the blood flow, a tiny incision was made in the external carotid artery, and a nylon guide filament (4 - 0) was inserted into the internal carotid artery, advanced 17-20 mm from the bifurcation of the common carotid artery to block the base of the middle cerebral artery. After 2 hours of ischemia, the filament was gently removed to allow reperfusion. The wound was sutured and the animal was put back into a new cage for 24 hours or 72 hours. The sham operation group exposed only the cervical artery isolations and did not undergo filament insertion. After the rats were awake, the neurological function was evaluated by Longa score, and the score of 2-4 was considered as successful occlusion of the MCAO. The neurological score was divided into 6 grades: 0 : normal, no neurological defect; 1: left forepaw could not be fully extended, mild neurological defect; 2: when walking, rats turned to the left (paralyzed side), moderate neurological deficit; 3: when walking, the 
body of the rat was dumped to the left (paralyzed side), severe neurological deficit; 4: unable to walk spontaneously, loss of consciousness; and 5: death.

\subsection{Detection of cerebral infarction area by TTC staining}

One day after ischemia-reperfusion, 4 rats in each group were randomly selected for euthanasia and brain extraction (on ice). The brains were placed in a refrigerator at $-20^{\circ} \mathrm{C}$ for quick frozen. The brains were then cut coronally to 5 slices with a thickness of about $2 \mathrm{~mm}$ each. These brain sections were incubated in $2 \%$ TTC phosphate buffer (PBS) for $30 \mathrm{~min}$ at $37^{\circ} \mathrm{C}$. The normal brain tissue was stained red, while the infarct area was white. The stained sections were fixed in $4 \%$ paraformaldehyde for 12 hours, and then scanned to a computer. The infarct areas were measured by ImageJ software, and the percentage of cerebral infarction area was calculated and presented as per hemisphere.

\subsection{HE staining}

The rat brains were fixed in $4 \%$ paraformaldehyde for 24 hours, dehydrated in accelerating concentrations of ethanol, cleared in xylene, embedded in paraffin, and sliced on a microtome with a thickness of $5 \mu \mathrm{m}$. The sections were stained with hematoxylin eosin (HE) and examined under a 400-fold optical microscope. Bright red eosinophils with shrinking triangular dense purple nuclei are considered necrotic cells. In each section, 5 microscopic fields of hippocampal CA1 region were selected to count the number of necrotic cells. The results were expressed as the percentage of necrotic cells in the total number of cells.

\subsection{Immunohistochemistry}

The endogenous peroxidase was blocked by $3 \% \mathrm{H} 2 \mathrm{O} 2$. The sections were rinsed with PBS for 10 min at room temperature. The sections were incubated with anti-p-S6 (dilution 1:100) antibody overnight at room temperature. On next day, the sections were rinsed with PBS and incubated with second anti-goat anti-rabbit IgG for $30 \mathrm{~min}$ at room temperature. Color reaction was achieved by incubating the sections with diaminobenzidine (DAB). The sections were counter stained with hematoxylin, mounted and observed under a light microscope.

\subsection{Western blotting detection}

The hippocampi were separated on ice bath, frozen in liquid nitrogen and stored in the refrigerator in $-80^{\circ} \mathrm{C}$. The tissues were homogenized and lysed with cell lysate buffer. The cytoplasmic and nuclear fractions were extracted by centrifugations. The protein concentrations were measured by BCA protein assay kit (Thermo Scientific). $25 \mu \mathrm{g}$ protein of each sample was loaded into $4 \% \sim 12 \%$ Nu-PAGE gel (Invitrogen, ThermoFisher) for electrophoresis. The protein was transferred to PVDF membrane (Millipore) and separately incubated overnight with primary antibodies against mTOR, p-mTOR, DRP1, p-DRP1, OPA1 and $\beta$-actin(all at the dilution of 1:1000, Cell Signaling Technology). The target protein bands and internal loaded control protein bands were scanned to LI-COR Bio-sciences Odyssey infrared fluorescence scanner (Lincoln, NE, USA) after being incubated with fluorescent second antibody for 1 hour. The fluorescence 
intensity of the band was measured and expressed as the ratio of the target protein band to that of the loaded control.

\subsection{Simple Wess automatic protein determination}

The hippocampal tissue lysates were loaded to Simple Wess machine (Protein Simple, USA) and processed according to the manufacturer's instruction. The primary antibodies used include anti-SIRT3, anti-NIX/BNIP3L, anti-COX IV, and anti- $\beta$-actin (all at the dilution of 1:1000, Cell Signaling Technology). After setting the parameters using Computer Compass for Simple Wess Program, uploading the reaction solutions and antibodies, and placing the disposable capillary, the program was run 3 hours and the band optical densities were read and recorded by the machine automatically.

\subsection{Statistical method}

The data were analyzed by SPSS19.0 statistical software. A value of $P<0.05$ was considered statistically significant. Data that conform to the normal distribution are represented as the mean \pm standard deviation (SD), the statistical significance of was determined with one-way analysis of variance (ANOVA) and followed by LSD-t test. The non-normal distribution data are represented as median (quartile), and the statistical significance of was determined with rank sum test.

\section{Results}

\subsection{Blood glucose levels}

SD rats whose blood glucose level $>16.7 \mathrm{mmol} / \mathrm{L}$ after 1 week of STZ injection were considered successful induction of diabetic models. There was no significant difference in blood glucose level between DM $(17.60 \pm 2.752)$ and DMR $(18.03 \pm 2.397)$ groups, suggesting that rapamycin did not affect the blood glucose level (Fig. 1a).

\subsection{Comparison of neurological function scores}

Neurological function was measured using Longa score after 1 day of MCAO. The score of DM1d group ( $2.378 \pm 07941)$ was significantly higher than that of NG1d group $(1.351 \pm 0.5877, p<0.01)$, while it showed a decrease after rapamycin pretreatment $(1.757 \pm 0.5480, p<0.01 \mathrm{vs}$. DM1d). The results suggested that hyperglycemia aggravated the neurological dysfunction induced by cerebral I/R injury, while rapamycin mitigated this trend (Fig. 1b).

\subsection{TTC Staining}

TTC staining was performed 1 day after I/R. The infarct volume of DM1d group ( $40.26 \% \pm-4.459 \%)$ was higher than that of NG1d group $(13.20 \% \pm 2.295 \%, p<0.01)$. Consistent with above results, rapamycin pretreatment significantly decreased the infarct volume after 1 day of I/R in DM group $(24.42 \% \pm 2.882 \%$, $p<0.01)$. The results suggested that hyperglycemia aggravated the tissue injury after $I / R$, while rapamycin pretreatment relieved the enhanced damage caused by hyperglycemia (Fig. 1c\&d). 


\subsection{HE staining}

The neuronal injury in the hippocampal CA1 area was observed. The neurons of sham group appeared in orderly arrangements, similar size, regular cell body and rare acidophilic neurons. The cell membrane was relatively complete, the cytoplasm was stained uniformly, the nuclear membrane was clear and complete, and uniform chromatin granules could be seen in neurons of sham group. While in NG1d group, cells showed necrosis, degeneration, disordered arrangement, nuclear pyknosis and ambiguous nuclear membrane. The neuronal damage in NG3d group was more severe than that in NG1d group $(p<0.05)$. In DM1d group, cells were found more degenerative and necrotic than in NG1d group $(p<0.05)$. Similarly, the changes of cell morphology were more obvious in DM3d group than that in NG3d group $(p<0.05)$. After pretreatment with rapamycin, cell degeneration and necrosis were significantly reduced in DMR1d and DMR3d groups ( $p<0.05$ vs. NG1d and NG3d, respectively) (Fig. 1e\&f).

\section{5 mTOR signaling}

The behavior of mTOR signaling was assessed by Western blotting of total mTOR and p-mTOR and immunohistochemistry of $\mathrm{p}$-S6. The western blotting results showed that there were no significant differences among all the groups in mTOR protein levels (Fig. 2a\&b). However, p-mTOR levels were significantly different among the groups. Thus, the $p-m T O R$ significantly increased after $3 \mathrm{~d}$ of I/R in NG group compared to Sham and NG1d $(p<0.05)$. Compared with NG group, p-mTOR increased in DM1d and further elevated in DM3d (Fig. 2a\&c; $p<0.05$ ). Rapamycin pretreatment profoundly suppressed the $p$ mTOR levels in diabetic ischemic animals at 1 and 3 days of reperfusion (Fig. 2a\&c; $p<0.05$ ). Consistently, the ratio of p-mTOR to mTOR exhibited the same trend (Fig. 2d).

Consistently, immunohistochemistry of mTOR downstream protein p-S6 revealed that the immunoreactivity of p-S6 in NG1d and NG3d groups increased comparted with the Sham group in the hippocampal CA1 neurons, as reflected by the brown precipitates $(p<0.05)$. Compared with NG groups, the expression of p-S6 in DM groups showed further elevation after $1 d$ and $3 d$ of $1 / R(p<0.05)$, while the elevation was inhibited after rapamycin intervention (Fig. 2e\&f; $p<0.05$ ).

\subsection{Mitochondrial dynamin related proteins}

Mitochondrial fission protein total DRP1, p-DRP1 and fusion protein OPA1 were detected in the hippocampus using Western blot. The total DRP1 levels were not changed among all the groups (Fig. 3a\&b). In contrast, the p-DRP1 levels increased after 1d and 3d of I/R in NG group compared to the Sham $(p<0.05)$. OPA1 increased mildly in NG1d $(p<0.05)$ and returned to baseline in NG3d. Compared with NG groups, the protein expression of p-DRP1 in DM groups further increased after $1 \mathrm{~d}$ and $3 \mathrm{~d}$ of $\mathrm{I} / \mathrm{R}$ $(p<0.05)$, while OPA1 in DM groups increased only mildly after $3 d$ of $I / R(p<0.05)$. Rapamycin treatment markedly decreased the levels of p-DRP1 and increased the levels of OPA1 in DM animals after 1 and 3d of $\mathrm{I} / \mathrm{R}(\mathrm{p}<0.05)$. These results suggested that high glucose enhanced mitochondrial fission and rapamycin prevented diabetes-induced mitochondrial dynamic imbalance (Fig. 3a-e). 


\subsection{SIRT3 and Nix/BNIP3L}

SIRT3 and Nix/BNIP3L was detected in the hippocampal tissue by Simple Wess technique. Compared with shame group, the expression of SIRT3 and Nix/BNIP3L increased after 1d and 3d of I/R in NG condition $(P<0.05)$. DM suppressed the levels of SIRT3 and Nix/BNIP3L after $1 \mathrm{~d}$ and $3 \mathrm{~d}$ of I/R compared to NG counterparts $(P<0.05)$. Rapamycin treatment markedly increased the levels of SIRT3 and moderately elevated Nix/BNIP3L compared with the DM counterparts $(p<0.05)$ (Fig. $\mathbf{3 f - h})$.

\section{Discussion}

The current study demonstrated that DM enhanced the impairment of neurobehavioral performance compared to NG after I/R as reflected by the higher deficit score in DM than that of the NG animals. The worsened neurological deficit in DM animals was associated with enlarged infarct volume and increased number of neural damage in the hippocampal CA1 area. Treatment by rapamycin to DM ischemic animals improved neurological functional performance and reduced infarct volume and neuronal death. Therefore, rapamycin could be considered as therapeutic drug for ischemic patients accompanied with diabetes mellitus.

Rapamycin and its derivatives (such as everolimus and tacrolimus) are specific inhibitors of m-TOR (Ruiz-Torres et al. 2018). Rapamycin binds to intracellular receptor FKBP12 and directly acts on FRB (fkbp-12-rapamycin binding) domain in mTOR, thereby inhibiting the activity of mTORC1 kinase (Sun et al. 2018). Whether activation of mTOR pathway is detrimental or beneficial to ischemic injury remains controversial. For example, activating PI3K/Akt/m-TOR by MiR-27 activator or Hsp90 silencing has been shown to protect rat brain from ischemia and reperfusion damage, and to suppress oxidative stress and inflammatory response. In contrary, other studies have also demonstrated that inhibition of mTOR reduced nerve injury and promoted neuronal survival (Zhang et al. 2020). These contradictory results suggest the importance of delicate balance of mTOR signaling pathway in cerebral ischemia. Although whether mTOR is beneficial to ischemic injury remains a debate, it is undeniable that rapamycin, its inhibitor, has dominantly shown a protective effect in many nervous system diseases (Brandt et al. 2018;Hwang et al. 2017; Liu et al. 2016;Yang et al. 2015;Yang et al. 2019). In this study, we have observed that DM significantly increased the levels of p-mTOR and p-S6 levels after I/R and the neuroprotective effect of rapamycin in DM animals was linked with suppression of p-mTOR and p-S6, supporting the concept that inhibition of $\mathrm{m}$-TOR signaling protects the brain from ischemic damage. This is consistent with the results we have previously reported in rats subjected to global ischemia under hyperglycemic condition and normoglycemic conditions (Liu et al. 2016;Yang et al. 2015). The mechanisms mediating the protective effect of rapamycin may include modulation of PI3K/Akt, MAPK/ERK1/2, and autophagy signaling (Yang et al. 2019) .

Mitochondria play an important role in promoting neural survival and improving neural functional recovery after ischemic stroke. As highly dynamic organelles, mitochondria undergo continuous fission and fusion to maintain mitochondrial function and cell activity. Mitochondrial fission contributes to 
removal of damaged and redundant mitochondria. Mitochondrial fission is considered as a prerequisite for mitochondrial phagocytosis. Mitochondrial fusion facilitates exchange of content between mitochondria and regulates mitochondrial metabolism (Huang et al. 2020). The balance between mitochondrial fission and fusion is regulated by a group of dynamic related large GTPases located in the outer membrane and inner membrane of the mitochondria. Fission is driven by DRP1 and Fis 1 ; while fusion is driven by OPA1, and Mfn1 and Mfn2 (Singh et al. 2017). It has been reported that cerebral ischemia suppresses the levels of fusion regulators OPA1 and Mfn2, and increases the levels of division regulators DRP1 and Fis1. Excessive fission results in mitochondrial rupture and fragmentation (Yoo and Jung 2018). Our results showed that DM further increased the levels of p-DRP1, suggesting increased probability of excessive mitochondrial fission by diabetic hyperglycemia. The small scale increase of OPA1 in DM rats after I/R may represent a nature response by which mitochondria try to combat the excessive fission. Rapamycin profoundly inhibited the levels of p-DRP1 and increased the level of OPA1, suggesting the imbalance of mitochondrial fission and fusion could be prevented by rapamycin. It is likely that hyperglycemia further tilts mitochondrial dynamic balance towards fission and rapamycin is capable to prevent or reverse this dynamic imbalance.

SIRT3 is a deacetylate enzyme containing a variety of amino acids. It is a member of sirtuins family and a redox enzyme. SIRT3 has been shown to decrease cell death and tissue damage through blockade of mitochondrial permeability transition pore formation through deacetylation of cyclophilin $\mathrm{D}$ in the mitochondrial matrix, to activate mitochondrial biogenesis through deacetylation of PGC-1a, to regulate cell metabolism by modulating AMPK (Yu et al. 2019), and to inhibit the activity of m-TOR (Wang et al. 2021). There has been very few study reports the expression of SIRT3 in diabetic ischemic models. In current study, SIRT3 increased after ischemia and reperfusion in NG animals. DM inhibited this ischemiacaused increase; while rapamycin abolished the inhibitory effect of DM on SIRT3, suggesting that SIRT3 plays a protective role after ischemic brain damage.

Nix/BNIP3L locates in the outer membrane of mitochondria. Studies have shown that BNIP3L deficiency significantly aggravates ischemic brain injury (Wu et al. 2021), suggesting that BNIP3L can protect nerve cells. In current study, compared with NG group, DM significantly reduced the protein level of Nix/BNIP3L, and rapamycin treatment partially reversed its decline, suggesting that Nix/BNIP3L may have a protective effect against ischemic injury.

In conclusion, diabetic hyperglycemia aggravated brain damage caused by MCAO. This enhanced damage was associated with activations of $\mathrm{p}-\mathrm{mTOR}$, autophagy, mitochondrial fission and suppressions of SIRT3 and Nix/BNIP3L. Rapamycin prevented the DM enhanced ischemic brain damage, suppressed pmTOR, rebalanced mitochondrial dynamics, and increased the levels of neural survival factors SIRT3 and Nix/BNIP3L.

\section{Declarations}

\section{Acknowledgments}


We acknowledge the National Natural Science Foundation of China [Grant number 81560226,81860250 and 82060237] and the Natural Science Foundation of Ningxia Hui Autonomous [Grant number 2021AAC03371] for funding this research.

\section{Funding}

This work was supported by National Natural Science Foundation of China [Grant number 81560226 , 81860250 and 82060237]; and Natural Science Foundation of Ningxia Hui Autonomous [Grant number 2021AAC03371].

\section{Competing Interests}

The authors have no relevant financial or non-financial interests to disclose.

\section{Author Contributions}

Xiao Yang, Changchun Hei and P. Andy Li conceived and designed the experiments. Changchun Hei, Yujia Zhou, Chenyang Zhang, Meiling Cao and Shilin Yuan performed the experiments. Changchun Hei, Yixin Qin, Xiao Yang and P. Andy Li analyzed the data. Xiao Yang, Changchun Hei and P. Andy Li wrote the manuscript.

\section{Data Availability}

The datasets used or analyzed during the current study are available from the corresponding author on reasonable request.

\section{Ethics approval}

All animal procedures were approved by the Animal Ethics Committee of NingXia Medical University and Use Committee (Animal license No. SCXK (Ning) 2015-0001) in line with the USA National Institutes of Health (NIH) guidelines.

\section{Consent to publish}

All authors declare consent to participate and consent for publication.

\section{References}


1. Brandt C, Hillmann P, Noack A et al (2018) The novel, catalytic mTORC1/2 inhibitor PQR620 and the $\mathrm{PI3K} / \mathrm{mTORC} 1 / 2$ inhibitor PQR530 effectively cross the blood-brain barrier and increase seizure threshold in a mouse model of chronic epilepsy. Neuropharmacology 140: 107-120. https://doi.org/ 10.1016/j.neuropharm.2018.08.002

2. Chen $Y$, Guo S, Tang $Y$ et al (2020) Mitochondrial Fusion and Fission in Neuronal Death Induced by Cerebral Ischemia-Reperfusion and Its Clinical Application: A Mini-Review. Medical science monitor : international medical journal of experimental and clinical research 26: e928651. https://doi.org/ $10.12659 / \mathrm{msm} .928651$

3. Collaborators GDal (2020) Global burden of 369 diseases and injuries in 204 countries and territories, 1990-2019: a systematic analysis for the Global Burden of Disease Study 2019. Lancet (London, England) 396: 1204-1222. https://doi.org/ 10.1016/s0140-6736(20)30925-9

4. Dong X, Guan F, Xu S et al (2018) Influence of blood glucose level on the prognosis of patients with diabetes mellitus complicated with ischemic stroke. Journal of research in medical sciences : the official journal of Isfahan University of Medical Sciences 23: 10. https://doi.org/ 10.4103/17351995.223951

5. Guo C, Sun L, Chen X et al (2013) Oxidative stress, mitochondrial damage and neurodegenerative diseases. Neural Regeneration Research 8: 2003-2014. https://doi.org/ 10.3969/j.issn.16735374.2013.21.009

6. He M, Zhang T, Fan Y et al (2020) Deletion of mitochondrial uncoupling protein 2 exacerbates mitophagy and cell apoptosis after cerebral ischemia and reperfusion injury in mice. International journal of medical sciences 17: 2869-2878. https://doi.org/ 10.7150/ijms.49849

7. Hei C, Liu P, Yang X et al (2017) Inhibition of mTOR signaling Confers Protection against Cerebral Ischemic Injury in Acute Hyperglycemic Rats. International journal of biological sciences 13: 878-887. https://doi.org/ 10.7150/ijbs.18976

8. Huang C, Yan S and Zhang Z (2020) Maintaining the balance of TDP-43, mitochondria, and autophagy: a promising therapeutic strategy for neurodegenerative diseases. Translational neurodegeneration 9: 40. https://doi.org/ 10.1186/s40035-020-00219-w

9. Hwang JY, Gertner M, Pontarelli F et al (2017) Global ischemia induces lysosomal-mediated degradation of mTOR and activation of autophagy in hippocampal neurons destined to die. Cell Death Differ 24: 317-329. 2016/12/10. https://doi.org/ 10.1038/cdd.2016.140

10. Li J, Quan K, Wang Y et al (2020) Effect of Stress Hyperglycemia on Neurological Deficit and Mortality in the Acute Ischemic Stroke People With and Without Diabetes. Frontiers in neurology 11: 576895. https://doi.org/ 10.3389/fneur.2020.576895

11. Li Y, Zhong W, Jiang Z et al (2019) New progress in the approaches for blood-brain barrier protection in acute ischemic stroke. Brain research bulletin 144: 46-57. https://doi.org/ 10.1016/j.brainresbull.2018.11.006

12. Liu P, Yang X, Hei C et al (2016) Rapamycin Reduced Ischemic Brain Damage in Diabetic Animals Is Associated with Suppressions of mTOR and ERK1/2 Signaling. Int J Biol Sci 12: 1032-1040. 
2016/08/05. https://doi.org/ 10.7150/ijbs.15624

13. Patnaik R, Tripathi AK and Dwivedi A (2019) Advancement in the Pathophysiology of Cerebral Stroke. Springer, Singapore

14. Rinkel L, Nguyen T, Guglielmi V et al (2020) High Admission Glucose Is Associated With Poor Outcome After Endovascular Treatment for Ischemic Stroke. Stroke; a journal of cerebral circulation 51: 3215-3223. https://doi.org/ 10.1161/strokeaha.120.029944

15. Rodriguez-Rodriguez AE, Donate-Correa J, Rovira J et al (2019) Inhibition of the mTOR pathway: A new mechanism of beta cell toxicity induced by tacrolimus. Am J Transplant 19: 3240-3249. 2019/06/04. https://doi.org/ 10.1111/ajt.15483

16. Ruiz-Torres V, Losada-Echeberría M, Herranz-López M et al (2018) New Mammalian Target of Rapamycin (mTOR) Modulators Derived from Natural Product Databases and Marine Extracts by Using Molecular Docking Techniques. Marine drugs 16. https://doi.org/ 10.3390/md16100385

17. Singh AK, Kashyap MP, Tripathi VK et al (2017) Neuroprotection Through Rapamycin-Induced Activation of Autophagy and PI3K/Akt1/mTOR/CREB Signaling Against Amyloid-beta-Induced Oxidative Stress, Synaptic/Neurotransmission Dysfunction, and Neurodegeneration in Adult Rats. Mol Neurobiol 54: 5815-5828. 2016/09/24. https://doi.org/ 10.1007/s12035-016-0129-3

18. Sun JJ, Yin XW, Liu HH et al (2018) Rapamycin inhibits ox-LDL-induced inflammation in human endothelial cells in vitro by inhibiting the mTORC2/PKC/c-Fos pathway. Acta Pharmacol Sin 39: 336344. 2017/10/27. https://doi.org/ 10.1038/aps.2017.102

19. Wang Y, Li X and Zhao F (2021) MCU-Dependent mROS Generation Regulates Cell Metabolism and Cell Death Modulated by the AMPK/PGC-1a/SIRT3 Signaling Pathway. Frontiers in medicine 8: 674986. https://doi.org/ 10.3389/fmed.2021.674986

20. Wu X, Zheng Y, Liu M et al (2021) BNIP3L/NIX degradation leads to mitophagy deficiency in ischemic brains. Autophagy 17: 1934-1946. https://doi.org/ 10.1080/15548627.2020.1802089

21. Yang X, Hei C, Liu P et al (2015) Inhibition of mTOR Pathway by Rapamycin Reduces Brain Damage in Rats Subjected to Transient Forebrain Ischemia. Int J Biol Sci 11: 1424-1435. 2015/12/19. https://doi.org/ 10.7150/ijbs.12930

22. Yang $X$, Hei C, Liu P et al (2019) Prevention of post-ischemic seizure by rapamycin is associated with deactivation of mTOR and ERK1/2 pathways in hyperglycemic rats. Biochemical and biophysical research communications 520: 47-53. https://doi.org/ 10.1016/j.bbrc.2019.09.096

23. Yang Y, Ma S, Ye Z et al (2020) MCM7 silencing promotes cutaneous melanoma cell autophagy and apoptosis by inactivating the AKT1/mTOR signaling pathway. Journal of cellular biochemistry 121: 1283-1294. https://doi.org/ 10.1002/jcb.29361

24. Yoo SM and Jung YK (2018) A Molecular Approach to Mitophagy and Mitochondrial Dynamics. Mol Cells 41: 18-26. 2018/01/27. https://doi.org/ 10.14348/molcells.2018.2277

25. Yu L, Dong $X$, Xue $X$ et al (2019) Naringenin improves mitochondrial function and reduces cardiac damage following ischemia-reperfusion injury: the role of the AMPK-SIRT3 signaling pathway. Food \& function 10: 2752-2765. https://doi.org/ 10.1039/c9fo00001a 
26. Zhang E, Chen Q, Wang J et al (2020) Protective role of microRNA-27a upregulation and HSP90 silencing against cerebral ischemia-reperfusion injury in rats by activating PI3K/AKT/mTOR signaling pathway. Int Immunopharmacol 86: 106635. 2020/07/08. https://doi.org/ 10.1016/j.intimp.2020.106635

\section{Figures}

\section{Figure 1}

a Preischemic blood glucose levels in the rats. Blood glucose levels were significantly elevated in DM, DMR compared with NG. Data are mean \pm SD. \#\#p<0.01 vs. NG. $\Delta \Delta p<0.01$ vs. NG b Comparison of neurological function scores of rats in each group after 1 day. Data are mean $\pm S D$. ${ }^{*} p<0.01$ vs. the sham group. \#\#p $<0.01$ vs. NG. $\Delta \Delta \mathrm{p}<0.01$ vs. DM $\mathbf{c}$ Brain slices stained by TTC $\mathbf{d}$ Brain infarct volume of each group was determined. Data are mean $\pm S D$. ${ }^{\star *} p<0.01$ vs. sham in the same treatment group. $\# \# p<0.01$ vs. NG. $\Delta \Delta p<0.01$ vs. DM e HE staining microphotographs of the CA1 areas in each group. Arrows indicate necrotic cells, bar $=20 \mathrm{um} \mathbf{f}$ Summarized percentage of neuronal death in the CA1 area. Data are mean $\pm S D$. ${ }^{*} p<0.01$ vs. sham in the same treatment group, $\# p<0.05$ and $\# \# p<0.01$ vs. NG at the same reperfusion endpoints. $\Delta p<0.05$ and $\Delta \Delta p<0.01$ vs. DM at the same reperfusion endpoints

\section{Figure 2}

mTOR signals a Representative western blot of mTOR and p-mTOR in hippocampal tissue b-d Summarized ratios of target protein band optical densities to loading control e Representative images of p-S6 in the hippocampus. Arrows indicate positive p-S6 staining which shows brown precipitates, bar = $20 \mu \mathrm{m} f$ Summary of optical density measurements of $p$-S6. Data are mean \pm SD. ${ }^{* *} p<0.01 \mathrm{vs}$. sham in the same treatment group, \#p $<0.05$ and \#\#p $<0.01$ vs. NG at the same reperfusion endpoints. $\Delta p<0.05$ and $\Delta \Delta p<0.01$ vs. DM at the same reperfusion endpoints

\section{Figure 3}

a Representative western blots of DRP1, p-DRP1 and OPA1 b-e Summarized ratios of target protein band optical densities to loading control f Representative western blots of SIRT3 and NIX/BNIP3L g\&h Summarized ratios of target protein band optical densities to loading control. Data are mean \pm SD. ${ }^{*} \mathrm{p}<$ 0.01 vs. sham in the same treatment group, \#p $<0.05$ and \#\#p $<0.01$ vs. NG at the same reperfusion endpoints. $\Delta p<0.05$ and $\Delta \Delta p<0.01 \mathrm{vs.} D M$ at the same reperfusion endpoints 